\title{
Disordered Mott-Hubbard Physics in Nanoparticle Solids: Transitions Driven by Disorder, Interactions, and Their Interplay
}

\author{
Davis Unruh,* Alberto Camjayi, Chase Hansen, Joel Bobadilla, Marcelo J. Rozenberg, \\ and Gergely T. Zimanyi
}

Cite This: https://dx.doi.org/10.1021/acs.nanolett.0c03141

Read Online

ACCESS |

Џlll Metrics \& More

回国 Article Recommendations

Supporting Information

ABSTRACT: We show that adapting the knowledge developed for the disordered Mott-Hubbard model to nanoparticle (NP) solids can deliver many very helpful new insights. We developed a hierarchical nanoparticle transport simulator (HINTS), which builds from localized states to describe the disorder-localized and Mott-localized phases of NP solids and the transitions out of these localized phases. We also studied the interplay between correlations and disorder in the corresponding multiorbital Hubbard model at and away from integer filling by dynamical mean field theory. This DMFT approach is complementary to HINTS, as it builds from the metallic phase of the NP solid. The mobility scenarios produced by the two methods are strikingly similar and account for the mobilities measured in NP solids.

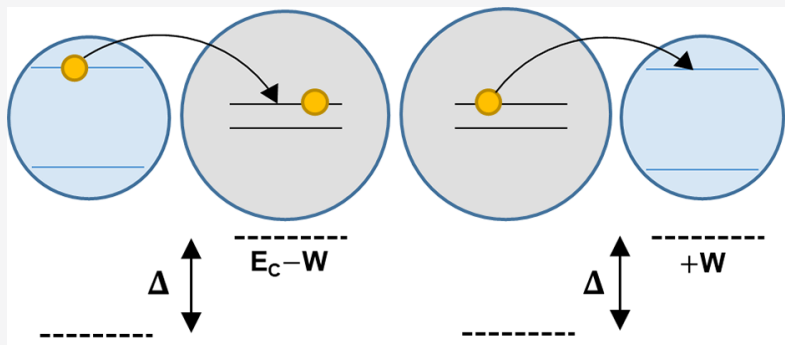

The mobility gap $\Delta$ due to the Coulomb blockade persists with increasing disorder $W$ We conclude this work by constructing the comprehensive phase diagram of PbSe NP solids on the disorder-filling plane.

KEYWORDS: Coulomb blockade, metal-insulator transition, Hubbard model, nanoparticles, Monte Carlo, dynamical mean field theory

$\mathrm{N}$ anoparticle (NP) solids are aggregates of nanometerscale particles with interesting and potentially useful emerging electronic functionalities. In NP solids, the wave functions are typically quantum confined to the NPs, making their electronic properties tunable with the NP size. ${ }^{1}$ This tunability makes them attractive for a wide variety of optoelectronic applications, ${ }^{2}$ including photovoltaics, ${ }^{3,4}$ lightemitting diodes, ${ }^{5}$ and field-effect transistors (FETs). ${ }^{6,7}$ However, the same quantum-confined localization also drives the NP solids to be insulating, hindering charge transport and thus their utility. Therefore, driving NP solids from their insulating phase across a metal-insulator transition (MIT) into a conducting, metallic phase is a top priority to boost their utility. Recent experimental attempts to cross the MIT included atomic layer deposition (ALD), ${ }^{8}$ substitutional percolation, ${ }^{9}$ chemical doping, ${ }^{10,11}$ and photodoping. ${ }^{12}$ ALD infilling with metal oxides has already enhanced mobilities above $7 \mathrm{~cm}^{2} /(\mathrm{V} \mathrm{s}){ }^{8}$ Whether these enhanced-mobility $\mathrm{NP}$ solids support coherent metallic transport is still debated. Building on these advances, NP solar cells were developed with impressive $13-16 \%$ power conversion efficiencies. ${ }^{13-15}$

There is vast literature on the theory of the disorder-driven, Anderson-type MIT of noninteracting electrons. ${ }^{16}$ The disorder of the site energies breaks up the site-to-site phase coherence of the originally extended wave functions, thereby localizing the electrons. Introducing interactions into Anderson localizaton makes the physical scenarios more complex, as revealed by scaling methods. ${ }^{17-19}$ Implications for transport were studied, e.g., via the concept of the Coulomb blockade/ gap, the energy cost of the attraction between the moving electron and the hole it leaves behind, thus suppressing transport at all fillings. ${ }^{20}$ These ideas were adapted to NP solids, e.g., in ref 10. Transport has been described as nearestneighbor hopping at high $T^{21,22}$ and as Efros-Shklovskii (ES) variable range hopping at low $T .^{23}$

Interactions are especially important at commensurate fillings. An electron already on an NP blocks the transport of additional electrons through that same NP because of the Coulomb cost of double occupancy. This was referred to as a Mott-gap or Coulomb blockade. This blockade fully blocks transport only at integer fillings. ${ }^{24-26}$

In recent years, the analysis of the interplay of disorder and interactions was re-energized by adaptating the dynamical mean field theory (DMFT) for analogous Hubbard models. $^{27-29}$ A surprising prediction of DMFT was that at $n=1$, at intermediate repulsion, increasing disorder first dissolved the Mott-localized phase into a metal, which then transformed into an Anderson-localized phase only at a higher disorder. Accordingly, the gap of the Mott-localized phase did not

Received: July 30, 2020

Revised: November 13, 2020 
persist into the Anderson-localized phase with increasing disorder. $^{28,29}$ Fillings with $n \neq 1$ have not yet been investigated with DMFT.

Remarkably, despite all this progress, profound unmet needs remain.

(1) In spite of inviting analogies, the vast amount of knowledge developed for the disordered Mott-Hubbard physics has not yet been adapted for NP solids, beyond some early suggestions. ${ }^{30-36}$ For example, even though the mobility of some NP solids exhibits maxima and minima as the filling is tuned, these features were not attributed to MottHubbard commensuration. ${ }^{37}$ Adapting Hubbard-based knowledge to nanomaterials could inspire new pathways to improve the transport properties of NP solids. In reverse, articulating these connections could make nanoparticle solids a rich and well-controlled testing ground for Hubbard-based research.

(2) We are not aware of a theory of the MIT that starts from the insulating phase either in NP solids or in the MottHubbard field. Notably, both the scaling and the DMFT techniques are built with extended wave functions and thus indicate Anderson/disorder localization only as the boundary where their applicability breaks down. Therefore, they are illsuited to describe the disorder-localized phase itself. Thus, developing a theory of the MIT out of the insulating phase would complement the MIT theories from the metallic phase, thus creating a comprehensive characterization of the MIT.

In response to these needs, here are the main messages of our paper. (1) We advocate that adapting disordered MottHubbard ideas for NP solids, and viewing NP solids as wellcontrolled experimental platforms for Mott-Hubbard models, provides extensive benefits for both fields. (2) To start this adaptation, we developed a hierarchical nanoparticle transport simulator to reach the MIT from the localized phase, and we analyzed a multiorbital Hubbard model with DMFT to reach the MIT from the delocalized phase. Using the combination of these complementary methods we determined the comprehensive phase diagram of nanoparticle solids that consists of two distinct localized phases defining two distinct MITs, which can be crossed by tuning various control parameters. (3) Tuning the filling $n$ toward integer values drives a disorderlocalized-to-Mott-localized transition. (4) For $n=1$ and large interactions, decreasing disorder drives a direct disorderlocalized-to-Mott-localized transition without an intervening metallic phase, characterized by a persistent gap. (5) For $n \neq 1$, decreasing disorder drives a disorder-localized-to-metal, Anderson-like MIT. (6) The DMFT-determined filling dependence of the mobility at low disorder shows striking similarities to that at high disorder, demonstrating the internal consistence of our analysis.

HINTS Method. Our hierarchical nanoparticle transport simulator HINTS is a kinetic Monte Carlo transport simulator that is extended by an additional metallic transport channel. This extension makes HINTS capable of reaching the MIT from the insulating phase. In an introductory analysis at generic fillings, we reported reaching the MIT and interpreted it as a quantum percolation transition. ${ }^{38}$ However, we did not connect this MIT to Mott-Hubbard phenomena, did not consider its interplay with Anderson localization, and did not analyze the model at commensurate fillings.

HINTS simulates NP solids by a multilevel hierarchical approach. We start with computing the electronic energy levels by using a parametrized band structure of individual PbSe NPs with diameters in the $3-8 \mathrm{~nm}$ range. ${ }^{39}$ On the next level, we account for the Coulomb interaction via the on-site charging energy $E_{C}$ :

$$
E_{\mathrm{C}}=n\left(\Sigma^{0}+\frac{(n-1)}{2} \Sigma\right)
$$

Here, $n$ is the number of electrons on the NP after the electron is added. $\Sigma^{0}$ is the total electrostatic energy cost of loading an electron onto a neutral NP, while $\Sigma$ is the energy cost of the Coulomb repulsion with the $(n-1)$ electrons already on the NP. We calculate $E_{C}$ with the hybrid empiricalperturbative method of ref 40 .

We proceed by generating a superlattice of PbSe NPs with diameters selected from a Gaussian distribution. We randomly fill this superlattice with electrons to reach the filling $n$ electron/NP. As described below, the electrons will move from these random initial NPs to the energetically favorable NPs once their dynamics are simulated.

In our extended HINTS, the NP-NP transition rates are either Miller-Abrahams phonon-assisted hoppings or nonactivated metallic transitions, depending on whether the energy difference between the initial and final states was larger or smaller than a hybridization energy:

$$
\Gamma_{i \rightarrow j}= \begin{cases}\nu g_{i j} \beta_{i j} \exp \left(\frac{-\Delta E_{i j}}{k_{b} T}\right) & \text { if } \Delta E_{i j} \geq \mathrm{E}_{\mathrm{H}} \\ \nu g_{i j} \beta_{i j} & \text { if } \Delta E_{i j}<\mathrm{E}_{\mathrm{H}}\end{cases}
$$

The attempt frequency $\nu$ is chosen to be $10^{12} \mathrm{~s}^{-1}$ to match experimental data; $g_{i j}$ is the product of the initial density of states on $\mathrm{NP}_{i}$ and the final density of states on $\mathrm{NP}_{j}$, and $\beta_{i j}$ is the WKB tunneling amplitude. The hybridization energy $E_{\mathrm{H}}$ is determined by the overlap of the electron wave functions of the NPs' $i$ and $j$. The energy difference between the initial and final configurations $\Delta E_{i j}$ is

$$
\Delta E_{i j}=\Delta E_{i j}^{s p}+\Delta E_{i j}^{F}+\Delta E_{i j}^{C}
$$

where $\Delta E_{i j}^{\mathrm{sp}}$ is the difference in single-particle band energies; $\Delta E_{i j}^{\mathrm{C}}$ is the difference in charging energies; and $\Delta E_{i j}^{\mathrm{F}}$ is the difference of the potential energy from the external electric field.

Finally, we compute the mobility of the NP solid by an extended kinetic Monte Carlo (KMC) algorithm with a sufficiently small voltage bias. In particular, we measure the mobility only after the transients, driven by the initial conditions, have decayed and the flow of electrons reached a steady state. See the Supporting Information for the full details of the HINTS method. We move to presenting the results of the HINTS simulations.

Disorder-localized-to-Mott-localized Transition with a Scan of the Filling $\boldsymbol{n}$. Experimentally, the electron/NP filling $n$ can be scanned by increasing the average NP diameter at a fixed volumetric charge density. Two experimental groups measured the dependence of the mobility on the NP diameter in NP-FETs and reported an initial rise followed by a maximum. ${ }^{37,41}$

We now use HINTS to compute the conductivity of PbSe NP solids as a function of NP diameter $(d)$. Figure 1 shows the HINTS-computed conductivities with increasing $d$ for two representations of the disorder, i.e., fixed-diameter disorder of $\pm 0.3 \mathrm{~nm}$ and variable-diameter disorder of $\pm 5 \%$. These results are overlaid on the experimental data of Kang et al. ${ }^{37}$ The 


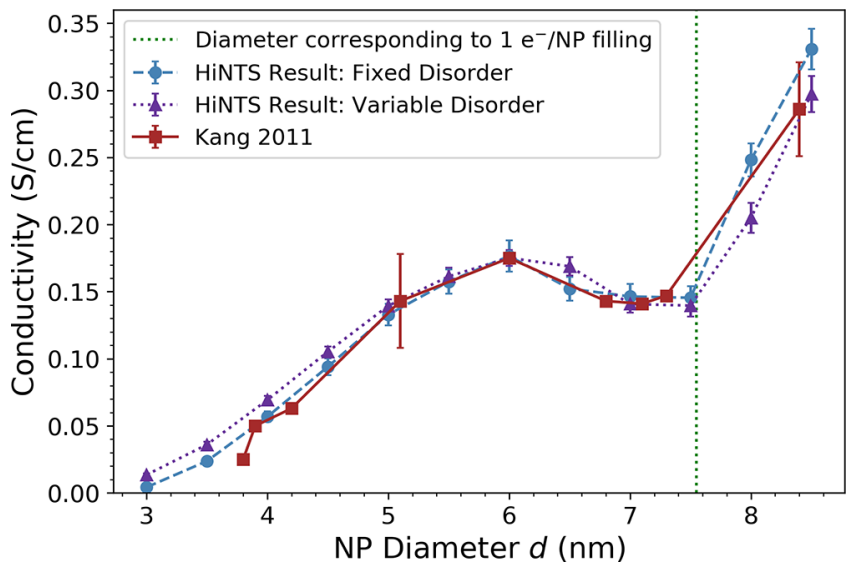

Figure 1. Conductivity vs NP diameter. HINTS simulations of PbSe NPs overlaid with experimental data. Volumetric electron density = $0.0016 \mathrm{e} / \mathrm{nm}^{3}$, ligand length $=0.5 \mathrm{~nm}$, and $T=200 \mathrm{~K}$.

HINTS conductivities and the Kang et al. data exhibit remarkable agreement.

The broad rising trend of the mobility/conductivity is driven by two factors, i.e., (i) a larger $d$ means that the electrons can traverse a fixed length by fewer hops, and (ii) since the electron energy-diameter relationship flattens with increasing $d$, the energy disorder induced by the diameter disorder decreases with increasing $d$.

The Kang experiments and our simulations both display a nonmonotonic pattern overlaid on this broad rising trend. Conspicuously, the conductivity maximum at $d=6 \mathrm{~nm}$ is observed to be only the beginning of a maximum-minimumresumed rise pattern, centered on $n=1$. The utility of adapting Mott-Hubbard ideas to analyze NP solids is compellingly demonstrated here by recognizing that such a conductivity minimum also arises when $n$ is scanned across $n=1$ in the repulsive Hubbard model, as the Coulomb repulsion opens a Mott gap at $n=1$, and thus suppresses transport through the occupied NPs.

For context, we mention that earlier experiments ${ }^{41}$ reported only a mobility maximum/plateau, which we reproduced by simulation. ${ }^{42}$ However, neither works observed the maximumminimum pattern and correspondingly did not recognize that Mott-Hubbard commensuration physics drives all these phenomena.

Changing $d$ also varies site energies and hopping rates and thus convolutes the transition into the Mott-localized phase with other trends. Therefore, next we isolate the mobility's dependence on $n$ in fixed-diameter NP solids. Experimentally this can be achieved by varying the FET gate voltage applied to a NP solid.

Figure 2 shows the HINTS-simulated mobility of a PbSe NP solid as $n$ is varied. Visibly, the mobility exhibits minima at integer fillings, accompanied by maxima close to half-integer fillings, just as with increasing $d$. These features are much more pronounced than in Figure 1.

The temperature dependence of the mobility/conductivity is activated for all fillings in Figures 1 and 2, having a smaller, disorder-induced gap away from $n=1$ that is boosted by the Coulomb blockade to a larger gap at $n=1$. Thus, scanning with $n$ through $n=1$ crosses from a disorder-localized phase into a Mott-localized phase and then back to a disorderlocalized phase for $n>1$, as expected from adapting the Hubbard model for these NP solids. And in reverse, the fact

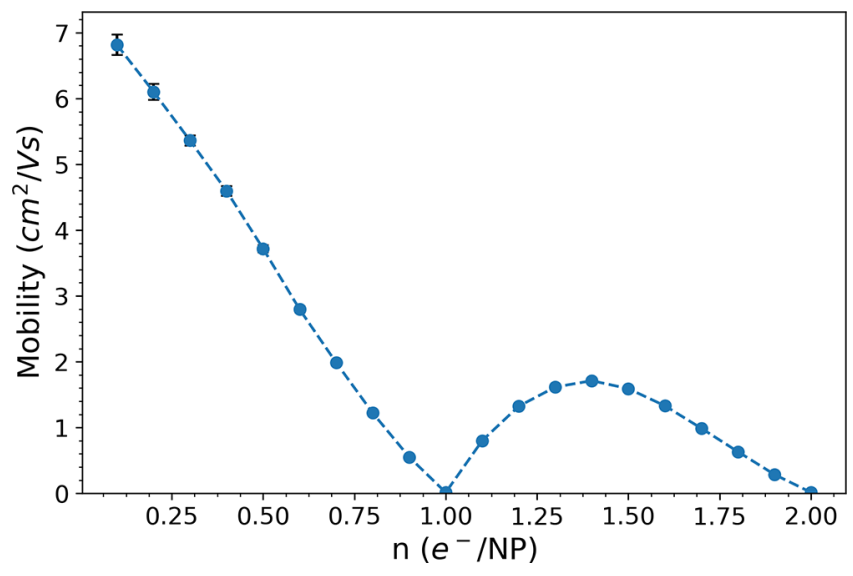

Figure 2. Electron mobility as a function of carrier filling in the disorder-localized phase of PbSe NPs with diameters of $6.6 \pm 0.3 \mathrm{~nm}$. $T=80 \mathrm{~K}$. Average charging energy $E_{\mathrm{C}}=100 \mathrm{meV}$, and the average hopping energy $t=7 \mathrm{meV}$.

that the experimental data and our simulations show such remarkable correspondences is compelling evidence that NP solids are well-controlled and tunable experimental realizations of the disordered Hubbard model.

Experimentally, such filling-driven disorder-localized-toMott-localized phase transitions have been reported in $\mathrm{Si}$ quantum dot arrays, where the gate voltage was used to tune the filling ${ }^{32}$ and in InAs quantum dot solids. ${ }^{43}$

Mott-localized-to-Disorder-localized Transition with a Scan of Disorder $\boldsymbol{W}$ at $\boldsymbol{n}=\mathbf{1}$. Next, we explore the robustness of the Mott-localized phase as the disorder $W / 2 t$ is varied at fillings around $n=1$. Here, the Hubbard $t$ kinetic energy was determined by mapping our tunneling probabilities to Fermi's golden rule. $W$ was determined from the sum of the disorder of the band and charging energies. We conduct this study on PbSe NP solids with a mean diameter of $6.5 \mathrm{~nm}$ by scanning the diameter dispersity up to $10 \%$.

Figure 3 shows the dependence of the activation energy/gap $\Delta$ on the disorder $W / 2 t$ at $n=1(\operatorname{scan} 3 \mathrm{a})$ and at $n=1+\delta$,

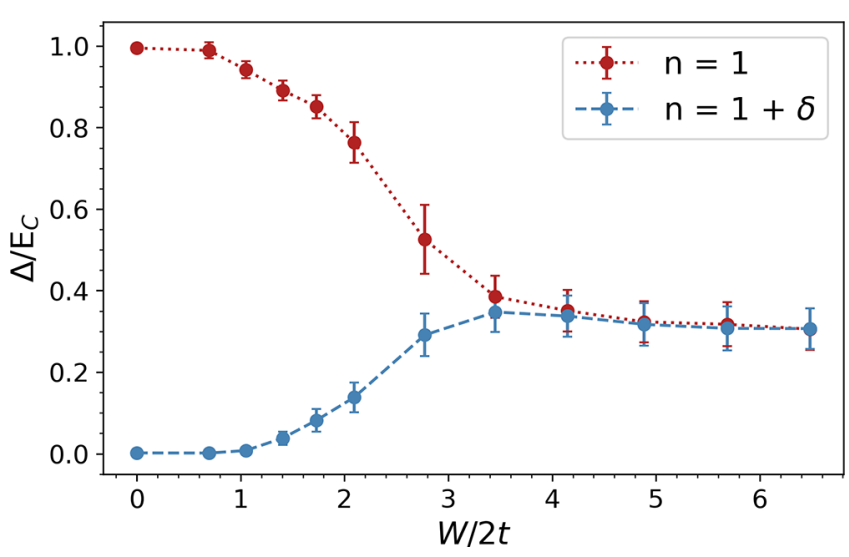

Figure 3. Disorder-dependent gap $\Delta(W)$ for PbSe NPs: (a) $n=1$, (b) $n=1+\delta . W_{\mathrm{c}}(\mathrm{Mott}) / 2 t \approx 3.5 ; W_{\mathrm{c}}(\mathrm{MIT}) / 2 t \approx 1.5$.

where $\delta=0.001$ (scan 3b). At $n=1$ and where the small disorder of the gap $\Delta$ is Mott-like, as its value is set by the charging energy $E_{\mathrm{C}}$, then $\Delta / E_{\mathrm{C}} \approx 1$. This Mott gap suppresses transport and creates a Mott-localized phase. With increasing disorder, the gap $\Delta$ gets re-normalized to a lower value $\Delta(W)$. 
The physics of this lower gap region is clarified by scan $3 b$ of $\Delta(W)$ at the filling $n=1+\delta$. The gap $\Delta(W)$ is the same for $n$ $=1$ and $n=1+\delta$ above a critical value $W_{\mathrm{c}}(\mathrm{Mott}) / 2 t \approx 3.5$. This insensitivity of the gap to the charging energy and to commensuration identifies this phase as a disorder-localized phase, into which the Mott-localized phase of $n=1$ transitions as $W$ exceeds $W_{\mathrm{c}}$ (Mott). Clearly, the transition at $n=1$ across $W_{c}$ (Mott) is direct, in the sense of having a persistent gap across the transition and no intervening gapless metallic phase. An analogous direct Mott-to-Anderson transition was reported by DMFT studies with a disorder scan in the Hubbard model at $n=1$ at around $W_{\mathrm{c}}(\mathrm{Mott})=3$, in good agrement with our result. Both studies worked in the $U / t=10-14$ range. $^{28}$ It is noteworthy that the gap is relatively insensive to $W$ in the disorder-localized phase. This is explained by the charging energy $E_{\mathrm{c}}$ screening the disorder $W$.

Beyond the remarkable disorder-driven Mott-localized-todisorder-localized transition at $n=1$, scan $3 \mathrm{~b}$ also reveals that as the disorder $W$ is reduced at $n=1+\delta$, the gap shrinks to zero at $W_{\mathrm{c}}(M I T) / 2 t \approx 1.5$. The vanishing of the gap indicates the disorder below which the wave functions become delocalized again. In this regime, the phases of the wafunctions become important, and the metallicity of transport is restored. HINTS does not track the phases of the electron wave functions and thus can describe the transport with decreasing disorder only down to $W=W_{c}(\mathrm{MIT})$ but cannot enter the metallic phase itself. We identify $W_{c}(\mathrm{MIT})$ as indicating a disorder-localized-to-metal transition, taking place at $n \neq 1$. This MIT at $W_{c}($ MIT) at $n=1+\delta$ is disorder driven and is therefore distinct from the interaction-driven Mott transition at $W=W_{\text {c }}$ (Mott) at $n=1$.

For context, we comment on the expectation that all electronic states are localized in 2D. This expectation, however, was demonstrated only in noninteracting systems. ${ }^{16}$ In interacting systems, the complex interplay of interactions and disorder has been shown to drive metal-insulator delocalization transitions starting from the extended phase even in 2D. ${ }^{19,44,45}$ Our results, building from the insulating phase, are consistent with and complement these claims.

These results have direct experimental relevance for NP solids beyond just PbSe NPs. As shown in ref 11, the authors developed strategies to cross the MIT and induce band-like transport with the high mobility of $27 \mathrm{~cm}^{2} /(\mathrm{V} \mathrm{s})$ in CdSe NP FETs by increasing the kinetic energy $t$ and decreasing the disorder $W$. The researchers ${ }^{11}$ increased the wave function overlap, and thus $t$, by switching to the compact ligand thiocynate and by annealing at the elevated temperatures of $T$ $=200-250{ }^{\circ} \mathrm{C}$. They eventually reached values of $t=6-8$ meV. They also reduced $W$ by doping the CdSe NP FETs with indium which filled up traps and thus reduced the trap-related disorder, eventually giving rise to an effective gap of $\Delta=6-7$ meV.

Our simulations provide a firm foundation for these strategies. To establish a quantitative correspondence, one would need to redo our PbSe simulation for CdSe NP solids to determine the additional disorder from polydispersity. Without the benefit of this calculation, we only make the qualitative observation that the $W / 2 t$ ratio where ref 11 reports an MIT is of the order 1 and is thus consistent with our $W_{\mathrm{c}}(\mathrm{MIT}) / 2 t=$ 1.5 .

An additional strategy emerges from our simulations, namely, the reduction of the charging energy $E_{C}$. We showed that $E_{\mathrm{C}}$ sets the Mott gap and screens the gap in the disorder- localized phase, and thus reducing $E_{\mathrm{C}}$ is yet another strategy to cross the MIT in NP solids.

Disordered-Metal-to-Mott-Localized Transition as a Function of $\boldsymbol{n}$. We already established that the physics of NP solids is analogous to a disordered Hubbard model whose sites represent the individual NPs. We take into account the 8-fold degeneracy of the electronic states of the $\mathrm{PbSe} \mathrm{NPs}^{46}$ by adopting a four orbital (labeled by $a, b=1,2,3,4)$ Hubbard model with diagonal disorder. ${ }^{47}$ The Hamiltonian reads:

$$
\begin{aligned}
H= & \sum_{\langle i, j\rangle, a, \sigma} t_{i j} d_{i, a, \sigma}^{\dagger} d_{j, a, \sigma}+\sum_{i, a, \sigma}\left(w_{i}-\mu\right) n_{i, a, \sigma} \\
& +\sum_{a} U n_{a, \uparrow} n_{a, \downarrow}+\sum_{a \neq b, \sigma, \sigma^{\prime}} U n_{a, \sigma} n_{b, \sigma^{\prime}}
\end{aligned}
$$

Here, $\langle i, j\rangle$ label nearest neighbor sites, $n_{i, a, \sigma}=d_{i, a, \sigma}^{\dagger} d_{i, a, \sigma}$ is the density of electrons of spin $\sigma$ in orbital $a$ on site $i, \mu$ is the chemical potential, $t_{i j}$ is the nearest neighbor hopping, and $U$ is the Coulomb repulsion. The disorder is introduced through the random site potential energy $w_{i}$, independent of orbital and spin index. For further details, see the Supporting Information.

The NP solid is on a regular lattice to start from a bona fide metallic state. We explore the combined effect of Coulomb repulsion and site energy disorder. We simulate PbSe NPs by associating the on-site Hubbard repulsion $U$ with the NP charging energy by taking $U=E_{\mathrm{C}}=100 \mathrm{meV}$ and the kinetic term with the NP-NP hopping amplitude of $t=7 \mathrm{meV}$, making $U / 2 t=7$. These parameters match those of Figure 2, and thus our Hubbard model is to be viewed as a quantitative modeling of the PbSe NP solid.

Since $t / U \ll 1$, the Hubbard model is in strong coupling. Therefore, we adopt the dynamical mean field theory (DMFT), extended to include disorder effects. ${ }^{27-29,48}$

The DMFT approach maps the original lattice model onto an auxiliary quantum impurity model supplemented with a selfconsistency condition. The quantum impurity problem is then tackled by numerical simulations that we performed using the continuous-time quantum Monte Carlo (CTQMC) method, ${ }^{49,50}$ described in ref 51 . That method samples a diagrammatic expansion of the partition function in powers of the impurity-bath hybridization. For simplicity, for the noninteracting electrons we adopt a semicircular density of states (DOS) of bandwidth $4 t$, since single-site DMFT is not sensitive to the shape of the noninteracting DOS. DMFT is exact in infinite dimensions, and it has been shown to remain a good approximation for lower dimensional systems whose physics is local, as is the case for the present Mott system. The disorder is introduced through a random site energy with a uniform distribution in the interval $[-W, W]$ (see SI for details).

Figure 4 shows the electron mobility and the chemical potential $\mu$ of this Hubbard model as a function of the filling $n$ for the clean and disordered cases, at $T / t=0.02$. In the clean case, $\mu$ shows a jump at $n=1$; this indicates the emergence of a Mott gap that localizes the electrons. ${ }^{47}$ This is a remarkable result, as our DMFT technique finds this Mott-localized phase at the band filling of $1 / 8$, whereas band structure calculations for $\mathrm{PbSe}$, or traditional Hubbard mean-field theories, would not find such a Mott insulator below the customary band filling of $1 / 2$. So, our DMFT work establishes PbSe as a Mott system, where correlation effects play out in 4-fold degenerate orbitals.

At finite temperatures, this Mott gap makes the mobility exhibit a minimum as the filling crosses $n=1$. Clearly, DMFT 


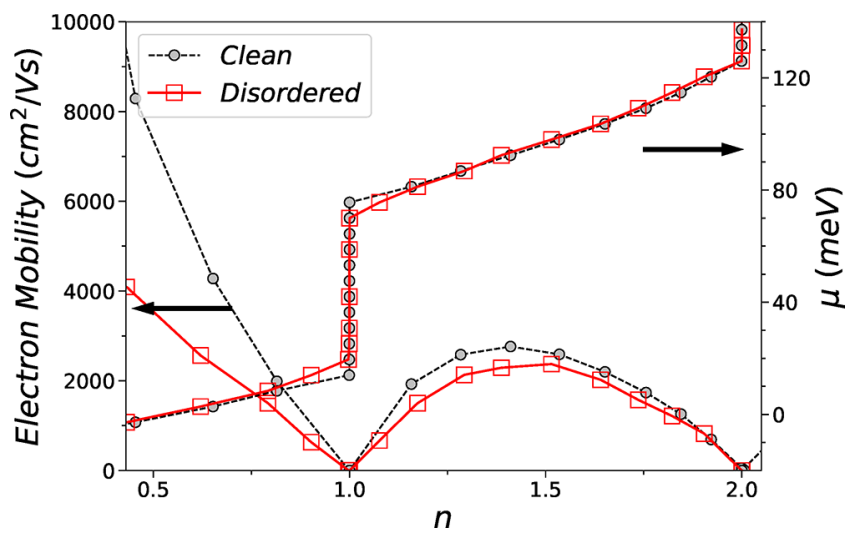

Figure 4. Mobility and chemical potential $\mu$ as filling scans from the metallic phase through the Mott gap.

established that the Mott gap/Coulomb blockade also suppresses the mobility in the metallic phase when the filling is scanned across $n=1$, just like in the insulating phase (cf. Figure 2).

A main result of the DMFT study is that the clean trends persist even for a substantial disorder $W / 2 t=1$. Indeed, the Mott gap (jump of $\mu$ ) at $n=1$ only decreases by a small amount and remains robust. This is reasonable since $W / 2 t=1$ $\ll W_{\mathrm{c}}($ Mott $) / 2 t=3.5$. At the same time, the mobility away from $n=1$ is reduced much more notably because the $W / 2 t=$ 1 disorder is relatively closer to the off-commensuration MIT at $W_{c}(\mathrm{MIT}) / 2 t=1.5$. Finally, Figure 4 shows that the relative reduction of the mobility by the disorder is strongest at small $n$. This makes physical sense, since at small $n$ the Fermi energy becomes comparable to the disorder, and thus, the relative importance of the disorder grows. To our knowledge, the present DMFT study is the first one done for a multiorbital model with disorder as a function of electron filling.

The here-used coherent potential CPA-DMFT method does not capture Anderson localization. While "typical medium" DMFT theories were proposed to capture a Mott-Anderson transition, this issue remains debated. ${ }^{52-59}$

\section{CONCLUSIONS}

We now bring together all the scans of our complementary HINTS and DMFT work and construct the phase diagram of $\mathrm{PbSe} \mathrm{NP}$ solids on the filling-disorder plane, shown in Figure 5. We distinguished a disorder-driven MIT at $n \neq 1$ and an interaction-driven MIT at $n=1$. In particular, at $n=1$, HINTS showed that at large interactions the Mott-localized-todisorder-localized transition occurs with a persistent gap. We complemented the studies building from the localized phases with studies building from the extended phase. Reassuringly, the complementary studies produced the same qualitative scenarios, as illustrated by the strikingly similar behavior of the mobility in Figures 2 and 4.

The totality of our studies demonstrated that adapting the vast body of knowledge developed for the disordered MottHubbard model for NP solids can and will produce many new insights into the physics of NP solids and, thus, can be used to develop strategies to improve their optoelectronic properties.

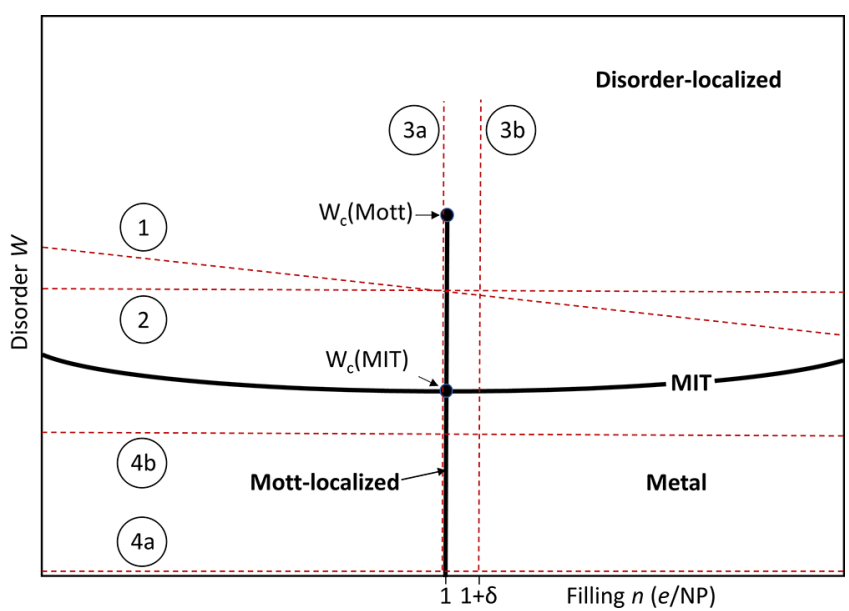

Figure 5. Qualitative phase diagram of NP solids in the (disorder $W-$ filling $n$ ) space, for $U \gg W$. The red dashed lines report the scans in the correspondingly labeled figures.

\section{ASSOCIATED CONTENT}

\section{(s) Supporting Information}

The Supporting Information is available free of charge at https://pubs.acs.org/doi/10.1021/acs.nanolett.0c03141.

Details of the HINTS and DMFT simulation methods (PDF)

\section{AUTHOR INFORMATION}

\section{Corresponding Author}

Davis Unruh - Physics Department, University of California, Davis, Davis, California 95616, United States; orcid.org/ 0000-0001-6992-9617; Email: dgunruh@ucdavis.edu

\section{Authors}

Alberto Camjayi - Departamento de Física, FCEyN, Universidad de Buenos Aires and IFIBA, Pabellón 1, Ciudad Universitaria, 1428 CABA, Argentina

Chase Hansen - Physics Department, University of California, Davis, Davis, California 95616, United States

Joel Bobadilla - Departamento de Física, FCEyN, Universidad de Buenos Aires and IFIBA, Pabellón 1, Ciudad Universitaria, 1428 CABA, Argentina

Marcelo J. Rozenberg - Laboratoire de Physique des Solides, UMR8502 CNRS - Université Paris-Sud, Université ParisSaclay, 91405 Orsay Cedex, France

Gergely T. Zimanyi - Physics Department, University of California, Davis, Davis, California 95616, United States

Complete contact information is available at:

https://pubs.acs.org/10.1021/acs.nanolett.0c03141

\section{Notes}

The authors declare no competing financial interest.

\section{ACKNOWLEDGMENTS}

We acknowledge helpful discussions with L. Qu, M. Voros, and M. Law. This work was supported by the UC Office of the President under the UC Laboratory Fees Research Program Collaborative Research and Training Award LFR-17-477148. A.C. and J.B. gratefully acknowledge support from CONICET and UBACyT. C.H., D.U., and G.T.Z. were supported by the National Science Foundation under award DMR-2005210. 


\section{REFERENCES}

(1) Kovalenko, M. V.; Manna, L.; Cabot, A.; Hens, Z.; Talapin, D. V.; Kagan, C. R.; Klimov, V. I.; Rogach, A. L.; Reiss, P.; et al. Prospects of Nanoscience with Nanocrystals. ACS Nano 2015, 9, 1012-1057.

(2) Talapin, D. V.; Lee, J.-S.; Kovalenko, M. V.; Shevchenko, E. V. Prospects of Colloidal Nanocrystals for Electronic and Optoelectronic Applications. Chem. Rev. 2010, 110, 389-458.

(3) Nozik, A. J. Quantum dot solar cells. Phys. E 2002, 14, 115-120.

(4) Kamat, P. V. Quantum Dot Solar Cells. Semiconductor Nanocrystals as Light Harvesters. J. Phys. Chem. C 2008, 112, 18737-18753.

(5) Shirasaki, Y.; Supran, G. J.; Bawendi, M. G.; Bulović, V. Emergence of colloidal quantum-dot light-emitting technologies. Nat. Photonics 2013, 7, 13-23.

(6) Talapin, D. V.; Murray, C. B. PbSe Nanocrystal Solids for n- and p-Channel Thin Film Field-Effect Transistors. Science 2005, 310, 8689.

(7) Hetsch, F.; Zhao, N.; Kershaw, S. V.; Rogach, A. L. Quantum dot field effect transistors. Mater. Today 2013, 16, 312-325.

(8) Liu, Y.; Tolentino, J.; Gibbs, M.; Ihly, R.; Perkins, C. L.; Liu, Y.; Crawford, N.; Hemminger, J. C.; Law, M. PbSe quantum dot fieldeffect transistors with air-stable electron mobilities above $7 \mathrm{~cm} 2 \mathrm{~V}-1$ s-1. Nano Lett. 2013, 13, 1578-1587.

(9) Cargnello, M.; Johnston-Peck, A. C.; Diroll, B. T.; Wong, E.; Datta, B.; Damodhar, D.; Doan-Nguyen, V. V.; Herzing, A. A.; Kagan, C. R.; Murray, C. B. Substitutional doping in nanocrystal superlattices. Nature 2015, 524, 450-453.

(10) Chen, T.; Reich, K. V.; Kramer, N. J.; Fu, H.; Kortshagen, U. R.; Shklovskii, B. I. Metal-insulator transition in films of doped semiconductor nanocrystals. Nat. Mater. 2016, 15, 299-303.

(11) Choi, J.-H.; Fafarman, A. T.; Oh, S. J.; Ko, D.-K.; Kim, D. K.; Diroll, B. T.; Muramoto, S.; Gillen, J. G.; Murray, C. B.; Kagan, C. R. Bandlike Transport in Strongly Coupled and Doped Quantum Dot Solids: A Route to High-Performance Thin-Film Electronics. Nano Lett. 2012, 12, 2631-2638.

(12) Talgorn, E.; Gao, Y.; Aerts, M.; Kunneman, L. T.; Schins, J. M.; Savenije, T.; van Huis, M. A.; van der Zant, H. S.; Houtepen, A. J.; Siebbeles, L. D. Unity quantum yield of photogenerated charges and band-like transport in quantum-dot solids. Nat. Nanotechnol. 2011, 6, 733-739.

(13) Lan, X.; Voznyy, O.; García de Arquer, F. P.; Liu, M.; Xu, J.; Proppe, A. H.; Walters, G.; Fan, F.; Tan, H.; Liu, M.; Yang, Z.; Hoogland, S.; Sargent, E. H. 10.6\% certified colloidal quantum dot solar cells via solvent-polarity-engineered halide passivation. Nano Lett. 2016, 16, 4630-4634.

(14) Jiao, S.; Du, J.; Du, Z.; Long, D.; Jiang, W.; Pan, Z.; Li, Y.; Zhong, X. Nitrogen-Doped Mesoporous Carbons as Counter Electrodes in Quantum Dot Sensitized Solar Cells with a Conversion Efficiency Exceeding 12\%. J. Phys. Chem. Lett. 2017, 8, 559-564.

(15) Sanehira, E. M.; Marshall, A. R.; Christians, J. A.; Harvey, S. P.; Ciesielski, P. N.; Wheeler, L. M.; Schulz, P.; Lin, L. Y.; Beard, M. C.; Luther, J. M. Enhanced mobility $\mathrm{CsPbI}_{3}$ quantum dot arrays for record-efficiency, high-voltage photovoltaic cells. Sci. Adv. 2017, 3, eaao4204.

(16) Lee, P. A.; Ramakrishnan, T. V. Disordered electronic systems. Rev. Mod. Phys. 1985, 57, 287-337.

(17) Finkel'shtein, A. M. Influence of Coulomb interaction on the properties of disordered metals. Zh. Eksp. Teor. Fiz. 1983, 84, 168189.

(18) Altshuler, B. L.; Aronov, A. G.; Lee, P. A. Interaction effects in disordered fermi systems in 2 dimensions. Phys. Rev. Lett. 1980, 44, $1288-1291$

(19) Castellani, C.; Kotliar, G.; Lee, P. A. Fermi-liquid theory of interacting disordered-systems and the scaling theory of the metalinsulator-transition. Phys. Rev. Lett. 1987, 59, 323-326.

(20) Schmid, G. Nanoparticles: From Theory to Application; John Wiley \& Sons: 2006.
(21) Yu, D.; Wang, C.; Wehrenberg, B. L.; Guyot-Sionnest, P. Variable Range Hopping Conduction in Semiconductor Nanocrystal Solids. Phys. Rev. Lett. 2004, 92, 216802.

(22) Liu, H.; Pourret, A.; Guyot-Sionnest, P. Mott and EfrosShklovskii Variable Range Hopping in CdSe Quantum Dots Films. ACS Nano 2010, 4, 5211-5216.

(23) Efros, A. L.; Shklovskii, B. I. Critical Behaviour of Conductivity and Dielectric Constant near the Metal-Non-Metal Transition Threshold. Phys. Status Solidi B 1976, 76, 475-485.

(24) Reich, K. V.; Chen, T.; Shklovskii, B. I. Theory of a field-effect transistor based on a semiconductor nanocrystal array. Phys. Rev. B: Condens. Matter Mater. Phys. 2014, 89, 235303.

(25) Chandler, R. E.; Houtepen, A. J.; Nelson, J.; Vanmaekelbergh, D. Electron transport in quantum dot solids: Monte Carlo simulations of the effects of shell filling, Coulomb repulsions, and site disorder. Phys. Rev. B: Condens. Matter Mater. Phys. 2007, 75, 085325.

(26) Qu, L.; Hansen, C.; Vörös, M.; Zimanyi, G. T. Commensuration effects in layered nanoparticle solids. Phys. Rev. B: Condens. Matter Mater. Phys. 2020, 101, 101.

(27) Aguiar, M. C. O.; Dobrosavljević, V.; Abrahams, E.; Kotliar, G. Critical Behavior at the Mott-Anderson Transition: A TypicalMedium Theory Perspective. Phys. Rev. Lett. 2009, 102, 156402.

(28) Bragança, H.; Aguiar, M. C. O.; Vučičević, J.; Tanasković, D.; Dobrosavljevic, V. Anderson localization effects near the Mott metalinsulator transition. Phys. Rev. B: Condens. Matter Mater. Phys. 2015, $92,125143$.

(29) Byczuk, K.; Hofstetter, W.; Vollhardt, D. Mott-Hubbard Transition versus Anderson Localization in Correlated Electron Systems with Disorder. Phys. Rev. Lett. 2005, 94, 056404.

(30) Sampaio; Beverly, K. C.; Heath, J. R. DC Transport in SelfAssembled 2D Layers of Ag Nanoparticles. J. Phys. Chem. B 2001, 105, 8797-8800.

(31) Byrnes, T.; Kim, N. Y.; Kusudo, K.; Yamamoto, Y. Quantum simulation of Fermi-Hubbard models in semiconductor quantum-dot arrays. Phys. Rev. B: Condens. Matter Mater. Phys. 2008, 78, 075320.

(32) Stafford, C. A.; Das Sarma, S. Collective Coulomb blockade in an array of quantum dots: A Mott-Hubbard approach. Phys. Rev. Lett. 1994, 72, 3590-3593.

(33) Stafford, C. A.; Das Sarma, S. Collective Coulomb blockade in an array of quantum dots: A Mott-Hubbard approach. Phys. Rev. Lett. 1994, 72, 3590-3593.

(34) Shabaev, A.; Efros, A. L.; Efros, A. L. Dark and photoconductivity in ordered array of nanocrystals. Nano Lett. 2013, 13, $5454-5461$.

(35) Remacle, F. On Electronic Properties of Assemblies of Quantum Nanodots. J. Phys. Chem. A 2000, 104, 4739-4747.

(36) Remacle, F.; Levine, R. D. Electronic Response of Assemblies of Designer Atoms: The Metal-Insulator Transition and the Role of Disorder. J. Am. Chem. Soc. 2000, 122, 4084-4091.

(37) Kang, M. S.; Sahu, A.; Norris, D. J.; Frisbie, C. D. Size- and Temperature-Dependent Charge Transport in PbSe Nanocrystal Thin Films. Nano Lett. 2011, 11, 3887-3892.

(38) Qu, L.; Vörös, M.; Zimanyi, G. T. Metal-Insulator Transition in Nanoparticle Solids: Insights from Kinetic Monte Carlo Simulations. Sci. Rep. 2017, 7, 7071.

(39) Kang, I.; Wise, F. W. Electronic structure and optical properties of $\mathrm{PbS}$ and $\mathrm{PbSe}$ quantum dots. J. Opt. Soc. Am. B 1997, 14, 16321646

(40) Delerue, C.; Lannoo, M. Nanostructures: Theory and Modeling; Springer-Verlag: Berlin, 2004.

(41) Liu, Y.; Gibbs, M.; Puthussery, J.; Gaik, S.; Ihly, R.; Hillhouse, H. W.; Law, M. Dependence of Carrier Mobility on Nanocrystal Size and Ligand Length in PbSe Nanocrystal Solids. Nano Lett. 2010, 10, $1960-1969$.

(42) Carbone, I.; Carter, S. A.; Zimanyi, G. T. Monte Carlo modeling of transport in PbSe nanocrystal films. J. Appl. Phys. 2013, 114, 193709.

(43) Kagan, C. R.; Murray, C. B. Charge transport in strongly coupled quantum dot solids. Nat. Nanotechnol. 2015, 10, 1013-1026. 
(44) Kravchenko, S. V.; Sarachik, M. P. Metal-insulator transition in two-dimensional electron systems. Rep. Prog. Phys. 2004, 67, 1-44.

(45) Castellani, C.; Di Castro, C.; Lee, P. A. Metallic phase and metal-insulator transition in two-dimensional electronic systems. Phys. Rev. B: Condens. Matter Mater. Phys. 1998, 57, R9381-R9384.

(46) An, J. M.; Franceschetti, A.; Dudiy, S. V.; Zunger, A. The Peculiar Electronic Structure of PbSe Quantum Dots. Nano Lett. 2006, 6, 2728-2735.

(47) Rozenberg, M. J. Integer-filling metal-insulator transitions in the degenerate Hubbard model. Phys. Rev. B: Condens. Matter Mater. Phys. 1997, 55, R4855-R4858.

(48) Georges, A.; Kotliar, G.; Krauth, W.; Rozenberg, M. Rev. Mod. Phys. 1996, 68, 13-125.

(49) Gull, E.; Millis, A. J.; Lichtenstein, A. I.; Rubtsov, A. N.; Troyer, M.; Werner, P. Rev. Mod. Phys. 2011, 83, 349-404.

(50) Haule, K. Quantum Monte Carlo impurity solver for cluster dynamical mean-field theory and electronic structure calculations with adjustable cluster base. Phys. Rev. B: Condens. Matter Mater. Phys. 2007, 75, 155113.

(51) Werner, P.; Gull, E.; Millis, A. J. Metal-insulator phase diagram and orbital selectivity in three-orbital models with rotationally invariant Hund coupling. Phys. Rev. B: Condens. Matter Mater. Phys. 2009, 79, 115119.

(52) Janiš, V.; Vollhardt, D. Coupling of quantum degrees of freedom in strongly interacting disordered electron systems. Phys. Rev. B: Condens. Matter Mater. Phys. 1992, 46, 15712-15715.

(53) Dobrosavljević, V.; Kotliar, G. Strong correlations and disorder in $\mathrm{d}=\infty$ and beyond. Phys. Rev. B: Condens. Matter Mater. Phys. 1994, 50, $1430-1449$.

(54) Ulmke, M.; Janiš, V.; Vollhardt, D. Anderson-Hubbard model in infinite dimensions. Phys. Rev. B: Condens. Matter Mater. Phys. 1995, 51, 10411-10426.

(55) Dobrosavljević, V.; Kotliar, G. Mean Field Theory of the MottAnderson Transition. Phys. Rev. Lett. 1997, 78, 3943-3946.

(56) Aguiar, M. C. O.; Dobrosavljević, V.; Abrahams, E.; Kotliar, G. Effects of disorder on the non-zero temperature Mott transition. Phys. Rev. B: Condens. Matter Mater. Phys. 2005, 71, 205115.

(57) Radonjić, M. c. v. M.; Tanasković, D.; Dobrosavljević, V.; Haule, K. Influence of disorder on incoherent transport near the Mott transition. Phys. Rev. B: Condens. Matter Mater. Phys. 2010, 81, 075118.

(58) Byczuk, K.; Hofstetter, W.; Vollhardt, D. Anderson Localization Vs. Mott-Hubbard Metal-Insulator Transition in Disordered, Interacting Lattice Fermion Systems. Int. J. Mod. Phys. B 2010, 24, $1727-1755$

(59) Bragança, H.; Aguiar, M. C. O.; Vučičević, J.; Tanasković, D.; Dobrosavljevic, V. Anderson localization effects near the Mott metalinsulator transition. Phys. Rev. B: Condens. Matter Mater. Phys. 2015, 92, 125143. 